\title{
Globe
}

Revue internationale d'études québécoises

\section{Dominique Marquis : Un quotidien pour l'Église. L'Action catholique, 1910-1940. Ottawa, Leméac, 2004}

\section{Cécile Vanderpelen-Diagre}

Volume 9, numéro 1, 2006

URI : https://id.erudit.org/iderudit/1000807ar

DOI : https://doi.org/10.7202/1000807ar

Aller au sommaire du numéro

Éditeur(s)

Globe, Revue internationale d'études québécoises

ISSN

1481-5869 (imprimé)

1923-8231 (numérique)

Découvrir la revue

Citer ce compte rendu

Vanderpelen-Diagre, C. (2006). Compte rendu de [Dominique Marquis : Un

quotidien pour l'Église. L'Action catholique, 1910-1940. Ottawa, Leméac, 2004].

Globe, 9(1), 280-283. https://doi.org/10.7202/1000807ar d'utilisation que vous pouvez consulter en ligne.

https://apropos.erudit.org/fr/usagers/politique-dutilisation/ 
Langevin, par la complexité discursive exemplaire de son déploiement de la figure, aura sans aucun doute un rôle clé à jouer dans une future histoire de la représentation de l'Autre au Québec.

Anthony Purdy

Université Western Ontario

\section{Dominique Marquis}

Un quotidien pour l'Église. L'Action catholique, 1910-1940.

Ottawa, Leméac, 2004.

Après l'incontournable essai sur l'Idéologie de L'Action catholique, réalisé par Richard Jones en 1974, il restait à décrire les conditions socioéconomiques de création, d'exploitation et de fonctionnement d'un journal qui, peu avant la Deuxième Guerre mondiale, connaissait un tirage de plus de 52000 exemplaires. C'est désormais chose faite grâce à l'ouvrage que Dominique Marquis a tiré de sa thèse de doctorat en histoire présentée à l'Université du Québec à Montréal. Pour ce faire, elle s'est appuyée sur de précieuses archives jusqu'à présent peu exploitées, à savoir les fonds de l'Action sociale catholique de l'archevêché de Québec, de la chancellerie de l'archevêché de Montréal et des Archives nationales du Québec, qui contiennent des dossiers sur L'Action catholique, et sur les papiers d'Eugène L'Heureux détenus par les Archives de l'Université Laval. Elle a également utilisé la base de données sur la presse québécoise Hyperbec, ce qui lui a permis de dresser des constats et comparaisons statistiques très utiles.

À partir d'une analyse minutieuse du contenu, de la mise en pages et de la stratégie publicitaire du quotidien, l'historienne décrit le processus suivi par l'Église pour participer à la culture de masse émergente. Habituée au journalisme de combat du xIX siècle, l'institution doit désormais compter sur un mode de communication différent, celui de la presse d'information du $\mathrm{xx}^{\mathrm{e}}$ siècle. S'adapter implique d'adopter un système et une pratique que les quotidiens concurrents - pour ne pas dire "adversaires" - manient avec efficacité. Bien que très réticents au début, 
les responsables de L'Action catbolique consentent progressivement à entrer dans une démarche de séduction à l'égard de leurs lecteurs, et à leur fournir des faits divers et des chroniques sportives substantielles. Les rubriques religieuses, à l'origine très présentes, voient leur nombre diminuer. La mise en pages évolue d'une manière drastique, et l'allure austère des débuts fait place dans les années 1930 à des titres attrayants, mis en valeur par des colonnes èt une police aérées, et, surtout, par des illustrations. Enfin, les responsables du journal doivent entrer dans le marché de la publicité, qui constitue pour le secteur un partenaire obligé. Ils ne s'autoriseront toutefois jamais à insérer des réclames pour des produits qu'ils réprouvent : le cinéma, le théâtre et, surtout, l'alcool. C'est là renoncer à un revenu très important. Pour combler ce vide, cependant, ils peuvent compter sur l'Action sociale catholique et l'archevêché, qui leur assurent des conditions financières convenables ainsi que des ressources humaines gratuites. "De la sollicitation directe aux grandes campagnes de propagande en passant par le denier de la presse, l'Église de Québec use de tous ses pouvoirs et de toutes ses influences pour assurer la survie du journal " (p. 185).

Mais l'Église et ses zélateurs ne sont pas les seuls pourvoyeurs du journal. Les contrats d'impression accordés par le gouvernement entre 1930 et 1950 montrent que l'installation au pouvoir de l'Union nationale de Duplessis coïncide avec une redistribution de ces contrats au profit de L'Action catholique et au détriment du Soleil, lequel bénéficiait jadis du quasi-monopole de cette lucrative rentrée.

Les relations du quotidien avec le secteur publicitaire, la hiérarchie ecclésiastique, les rouages de l'Action sociale catholique et le monde journalistique sont très bien analysés par l'auteure. Pour mettre en lumière cette dernière relation, des instruments de comparaison habiles avec Le Soleil et La Presse (type et nombre de chroniques, tirage, mise en pages, publicités, etc.) sont proposés. En revanche, l'analyse du contenu du journal aurait mérité d'être plus poussée. Peut-être l'était-elle dans la thèse? Le livre est en effet particulièrement synthétique puisqu'il condense en 220 pages une dissertation qui en faisait 435 .

La matière est pourtant des plus intéressantes. Au fil de l'histoire de L'Action catholique, se perçoit le passage de l'Église d'une action exclusivement pastorale à une présence dans tous les domaines du monde temporel. Si la cellule pastorale n'imposait pas à l'institution de sortir du 


\section{REVUE INTERNATIONALE D'ÉTUDES QUÉBÉCOISES}

cadre strictement socio-religieux de la vie de ses ouailles, l'industrialisation et l'alphabétisation l'obligent à délaisser ses missions traditionnelles. Désormais avides de divertissements, disposant de plus en plus de temps pour leurs loisirs, les nouveaux citadins ont des désirs neufs à satisfaire. Ainsi, dans tous les pays industrialisés où il s'est installé, le clergé investit-de nouveaux secteurs d'activités. Le plus bel exemple de cette démarche est sans doute le sport. Si, à la veille de la guerre, L'Action catbolique consacre à cette matière près de $12 \%$ de ses pages (p. 123), c'est certes pour séduire ses lecteurs, comme le propose Dominique Marquis (p. 98 et 123), mais c'est aussi parce que le sport est devenu un lieu de socialisation religieuse, c'est-à-dire un outil de prosélytisme. La résistance de l'Église à l'égard du corps est progressivement remplacée par un intérêt, puis par un investissement massif. Les mouvements de jeunesse, qui ont résolument intégré les habitudes sociales de l'entre-deux-guerres dans la plupart des pays industrialisés, ont imposé la pratique d'une activité physique et la recherche d'un corps sain. Ce phénomène a été très bien étudié en Frances. Qu'en est-il au Québec? La manière dont L'Action catbolique traite la rubrique sportive aurait pu donner lieu à d'intéressantes découvertes sur cette question, décidément bien présente. On pense entre autres à la figure du prêtre catholique qui, dans le célèbre roman $A u$ pied de la pente douce de Roger Lemelin, est incité à participer à un match pour reconquérir ses paroissiens séduits par un pasteur protestant fringant et sportif.

De même, il aurait été utile d'analyser les pages féminines. Comment le lectorat y est-il traité ? De quoi l'entretient-on? Qui s'occupe de ces pages? Les rubriques nous apprennent-elles des choses sur la vie de la femme catholique "modèle" et sur les mouvements catholiques féminins?

5. Bernard Dubreuil a consacré sa thèse de doctorat au sujet : " Les catholiques et les activités physiques *, thèse de doctorat, 3 tomes, Nice, 1998. Voir également son article * La naissance du sport catholique *, Alain EHRENBERG [éd.], Aimez-vous les stades, les origines bistoriques des politiques sportives en France, 1870-1930, Recherches, $\mathrm{n}^{\circ}$ 43, avril 1980, p. 221-251; Bruno Dumons et Gilles POLLET, "Églises chrétiennes et sport international dans la première moitié du $\mathrm{xx}^{e}$ siècle ", Alfred W $\mathrm{W}_{\mathrm{AHL}}$ [éd.], Jeux et sports dans l'bistoire, Actes du colloque de Metz, tome 1, C.T.H.S., 1986, p. 205-218; et Michel LAGREE, "Sport et sociabilité catholique en France au début du $\mathrm{XX}^{\mathrm{e}}$ siècle ", P. ARnaud et J. CAMY [éd.], La naissance du mouvement sportif associatif en France, Presses universitaire de Lyon, Lyon, 1986, p. 327-337. 
Enfin, on regrette qu'aucune place ne soit laissée aux pages culturelles. Évoluent-elles? Comment, par exemple, L'Action catholique perçoit-elle la rencontre de l'Église avec le monde du théâtre initiée par le père Legault? Quelle lecture fait-elle des romans édités au Québec? Son silence comme ses critiques sur ces sujets auraient mérité quelques mots. En ce qui concerne la participation croissante de l'Église à toutes les activités socioculturelles, une comparaison avec des organes de presse catholiques d'autres pays pourrait également s'avérer très intéressante.

Le dernier chapitre du livre se concentre sur l'équipe rédactionnelle du journal. La biographie collective présentée montre l'importance du réseau mobilisé et la professionnalisation progressive du noyau de base. L'intérêt du sujet aurait mérité, ici encore, quelque développement. Cette galerie de portraits manque décidément de chair, de vie. On termine ce chapitre en se demandant : que représentait dans la vie d'un homme ou d'une femme - le fait d'être journaliste pour L'Action catholique? Était-ce un poste sans avenir, un tremplin, un travail journalistique comme tout autre ? Remarquons, à cet égard, que la question de l'image du journal n'est pas abordée. Est-ce que, dans les mémoires et la correspondance, les contemporains parlent de ce quotidien? S'il n'est pas pensable de cerner d'une manière définitive le lectorat d'un journal, il est en revanche possible d'évaluer le degré d'impact sur ce dernier. La question se pose : quelle trace L'Action catbolique a-elle laissée dans la mémoire collective des Québécois?

Toutes ces questions montrent l'intérêt que suscite l'ouvrage de Dominique Marquis. Loin d'épuiser les interrogations, il les stimule, et c'est là un très grand mérite. Un autre de ses mérites est une présentation très claire, tant dans le propos que dans le découpage de l'argumentaire. Il est à souhaiter que l'historienne poursuive ses travaux et continue à contribuer à l'approfondissement de l'histoire de la rencontre entre religion et modernité au $x^{e}$ siècle, chantier d'autant plus intéressant qu'il concerne une brûlante actualité.

Cécile Vanderpelen-Diagre FNRS - Université libre de Bruxelles 